

\title{
Strong normal-incidence infrared absorption in self-organized InAs/InAlAs quantum dots grown on $\operatorname{InP}(001)$
}

A. Weber, Olivier Gauthier-Lafaye, F.H. Julien, Julien Brault, Michel Gendry, Y. Desieres, T. Benyatou

\section{To cite this version:}

A. Weber, Olivier Gauthier-Lafaye, F.H. Julien, Julien Brault, Michel Gendry, et al.. Strong normalincidence infrared absorption in self-organized InAs/InAlAs quantum dots grown on $\operatorname{InP}(001)$. Applied Physics Letters, 1999, 74 (3), pp.413-415. 10.1063/1.123045 . hal-02111140

\author{
HAL Id: hal-02111140 \\ https://hal.science/hal-02111140
}

Submitted on 25 Apr 2019

HAL is a multi-disciplinary open access archive for the deposit and dissemination of scientific research documents, whether they are published or not. The documents may come from teaching and research institutions in France or abroad, or from public or private research centers.
L'archive ouverte pluridisciplinaire HAL, est destinée au dépôt et à la diffusion de documents scientifiques de niveau recherche, publiés ou non, émanant des établissements d'enseignement et de recherche français ou étrangers, des laboratoires publics ou privés.

$$
\text { Copyright }
$$




\title{
Strong normal-incidence infrared absorption in self-organized InAs/InAlAs quantum dots grown on $\operatorname{InP}(001)$
}

\author{
A. Weber, O. Gauthier-Lafaye, and F. H. Julien ${ }^{\text {a) }}$ \\ Institut d'Electronique Fondamentale, URA CNRS 22, Université Paris-XI, 91405 Orsay, France \\ J. Brault and M. Gendry \\ Laboratoire d'Electronique-LEAME, UMR CNRS 5512, Ecole Centrale de Lyon, 69131 Ecully, France \\ Y. Désieres and T. Benyattou \\ Laboratoire de Physique de la Matière-LPM, INSA de Lyon, 69621 Villeurbanne Cedex, France
}

(Received 6 July 1998; accepted for publication 14 November 1998)

\begin{abstract}
InAs self-assembled quantum dots in InAlAs matrix grown on $\operatorname{InP}(001)$ substrates have been fabricated using Stranski-Krastanov growth mode. A strong in-plane polarized intraband absorption in the 10.6-20 $\mu \mathrm{m}$ wavelength region has been observed and ascribed to a transition from the ground electron state to an excited state confined in the layer plane along the [110] direction. The absorption at normal-incidence reaches $7.8 \%$ for ten layers of $n$-doped quantum dots. The oscillator strength of the intraband transition is comparable to that achieved in quantum wells for a conduction band intersubband transition. The dependence of the intraband absorption on carrier concentration and temperature suggests a quantum-wire type confinement potential. (C) 1999 American Institute of Physics. [S0003-6951(99)04803-2]
\end{abstract}

The self organization of strained semiconductor films obtained by epitaxial techniques is a powerful method to achieve a large concentration of low-dimensional nanostructures such as quantum dots or quantum wires. ${ }^{1}$ With respect to quantum wells, the additional in-plane confinement of carriers as well as the peaked density of states leads to attractive properties in the long-wavelength infrared region. This spectral domain corresponds to intraband transitions between confined states belonging to either the conduction or the valence band. Intraband spectroscopy has been shown to be a sound technique for investigating low-dimensional semiconductor structures because it provides a direct measurement of the confinement energies and of the spatial symmetry of the envelope wave functions. ${ }^{2,3}$ Intraband transitions are also of great interest for infrared applications such as photodetectors or unipolar lasers. ${ }^{4,5}$ In contrast to quantum wells, the inplane confinement of carriers in one-dimensional (1D) or zero-dimensional (0D) structures provides room for intraband transitions polarized in the plane of the layers which makes excitation at normal-incidence possible. This property is very attractive for photodetection applications, provided that large absorption quantum efficiencies can be obtained in order to achieve large detectivities. ${ }^{6}$ Normal-incidence intraband absorption has been recently reported in $n$-doped InGaAs/GaAs and InAs/AlGaAs quantum dots using photoconduction spectroscopy. ${ }^{7,8}$ However, because photoconductive gain may occur, direct absorption measurements are required to assess the actual absorption strength.

In this letter, we have investigated self-organized InAs $/ \mathrm{In}_{0.52} \mathrm{Al}_{0.48} \mathrm{As}$ quantum dots grown on $\operatorname{InP}(001)$ substrate using molecular beam epitaxy. We show that the $3 \%$ lattice mismatch characteristic of this material system, which is twice smaller than that of the well-studied InAs/GaAs sys-

${ }^{\text {a)} E l e c t r o n i c ~ m a i l: ~ j u j u @ ~ @ i e f . u-p s u d . f r ~}$ tem, allows full coverage of the surface with aligned and elongated quantum dots. The quantum dots have been characterized by atomic force microscopy (AFM), photoluminescence and infrared spectroscopy. We report on a large quantum efficiency intraband absorption at normal incidence in the 10.6-20 $\mu \mathrm{m}$ wavelength region. The intraband absorption is shown to result from an electron transition from the ground state to an excited state confined in the layer plane, perpendicularly to the quantum dot alignment axis. The dependence of the intraband absorption on carrier concentration and temperature suggests a quantum-wire type confinement potential.

The four samples studied in this work were grown at $525^{\circ} \mathrm{C}$ on a semi-insulating $\mathrm{InP}$ substrate using solid source molecular beam epitaxy. Sample A is a nonintentionally doped (n.i.d.) and contains one plane of InAs quantum dots. It consists of a $0.4 \mu \mathrm{m}$ thick $\operatorname{In}_{0.52} \mathrm{Al}_{0.48} \mathrm{As}$ buffer layer followed by $0.9 \mathrm{~nm}$ of InAs. The InAs thickness of 3 monolayers (ML) is just above the 2D/3D growth mode transition detected by reflection high electron energy diffraction at 2.5 ML. The InAs growth rate was $0.22 \mathrm{ML} / \mathrm{s}$ and the V/III beam equivalent pressure ratio was equal to 35 . The growth is then interrupted and the sample is maintained $120 \mathrm{~s}$ at $525^{\circ} \mathrm{C}$ under a $5 \times 10^{-6}$ Torr arsenic pressure. A $0.3 \mu \mathrm{m}$ thick $\operatorname{In}_{0.52} \mathrm{Al}_{0.48} \mathrm{As}$ cap layer is then deposited. Samples B, $\mathrm{C}$, and D contain ten planes of InAs quantum dots separated by $50 \mathrm{~nm}$ thick $\mathrm{In}_{0.52} \mathrm{Al}_{0.48} \mathrm{As}$ barriers. Sample B is $p$ doped with Be while samples $\mathrm{C}$ and D are $n$ doped with Si. Delta doping of the InAlAs barriers is performed $2 \mathrm{~nm}$ below each InAs quantum dot layer in order to achieve a sheet carrier density of $5 \times 10^{11} \mathrm{~cm}^{-2}$ for samples $\mathrm{B}$ and $\mathrm{C}$ and $2.5 \times 10^{11} \mathrm{~cm}^{-2}$ for sample D.

The top-right inset of Fig. 1 shows an AFM image of a typical uncapped n.i.d. InAs/InAlAs structure. As seen, the surface is almost fully covered with InAs elongated islands 


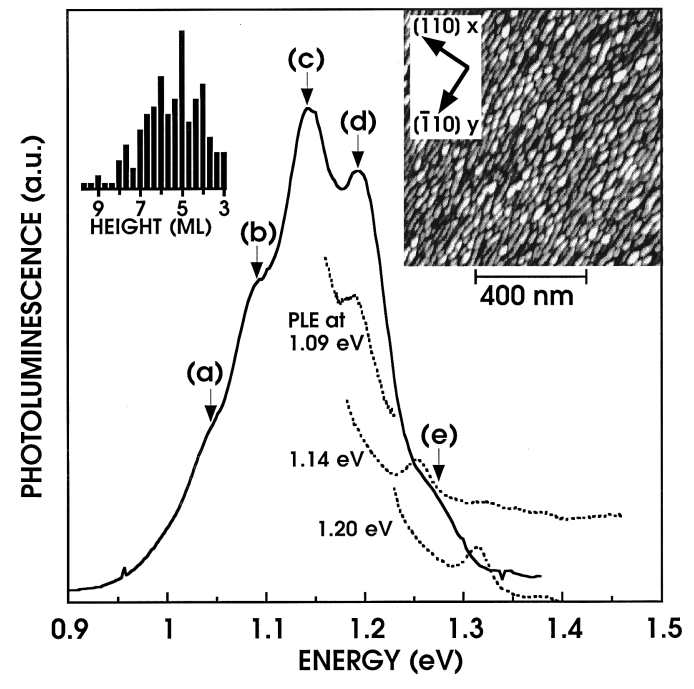

FIG. 1. Photoluminescence of the n.i.d. sample at $4.2 \mathrm{~K}$ under $15 \mathrm{~W} \mathrm{~cm}^{-2}$ excitation by an $\mathrm{Ar}^{++}$laser (full curve). The dotted curves which are vertically offset for clarity, are photoluminescence excitation spectra with the detection photon energy set at peaks (b), (c), and (d), respectively. The top-right inset shows an AFM image of a typical uncapped sample. The top-left inset is a histogram of the dot peak height in monolayers $(\approx 0.3 \mathrm{~nm})$ of a $0.5 \times 0.5 \mu \mathrm{m}^{2}$ region of the AFM image.

aligned in the $[\overline{1} 10]$ direction. The island density is of the order of $7 \times 10^{10} \mathrm{~cm}^{-2}$. The size of the islands deduced from AFM measurements is $56 \pm 11 \mathrm{~nm}$ along [ $[110]$ ( $y$ axis), $31 \pm 6 \mathrm{~nm}$ along [110] ( $x$ axis). The histogram of dot peak heights shown in the top-left inset of Fig. 1 reveals a broad distribution of sizes centered at $1.5 \mathrm{~nm}$ with a full width at half maximum (FWHM) of $0.6 \mathrm{~nm}$. The histogram shows clear accumulation of dots with height corresponding to an integer number of monolayers, 4,5 , and 6 , respectively. Note that the island shape and size could be modified after the deposition of the InAlAs capping layer because of element III exchanges between the islands and the matrix. However, a strong modification is not expected since such exchange is unfavorable for InAs/InAlAs from an alloy thermodynamical point of view. ${ }^{9}$

The photoluminescence (PL) spectrum at $4 \mathrm{~K}$ of sample A is shown in Fig. 1. A structured PL band is detected between 0.9 and $1.4 \mathrm{eV}$. The spectrum can be fitted with five Gaussian peaks labeled (a) to (e) with FWHM $\approx 50 \mathrm{meV}$. The low-energy side of the PL band arises from the larger dots. Based on AFM measurements, peaks (b), (c), and (d) are likely to arise from integer monolayer thickness fluctuations of the dots with heights close to 6,5 , and 4 monolayers, respectively. ${ }^{10}$ Peak (e) is ascribed to the excited state PL in addition to some ground state PL from smaller dots. This is confirmed by separate PL experiments at larger excitation intensities which reveal an increase of the relative intensity of peak (e) as a result of band filling of the excited states. ${ }^{11}$ Photoluminescence excitation spectroscopy (PLE) of dots (b), (c), and (d) reveals one excited state absorption peak which is shifted to higher energy from the ground state luminescence by 101,109 , and $120 \mathrm{meV}$, respectively. Note that these values reflect both the electron and heavy-hole quantum confinement and that the larger shifts correspond to smaller dots.

Infrared spectroscopy of the n.i.d. sample was performed Downloaded 04 Feb 2003 to 156.18.34.206. Redistribution subject

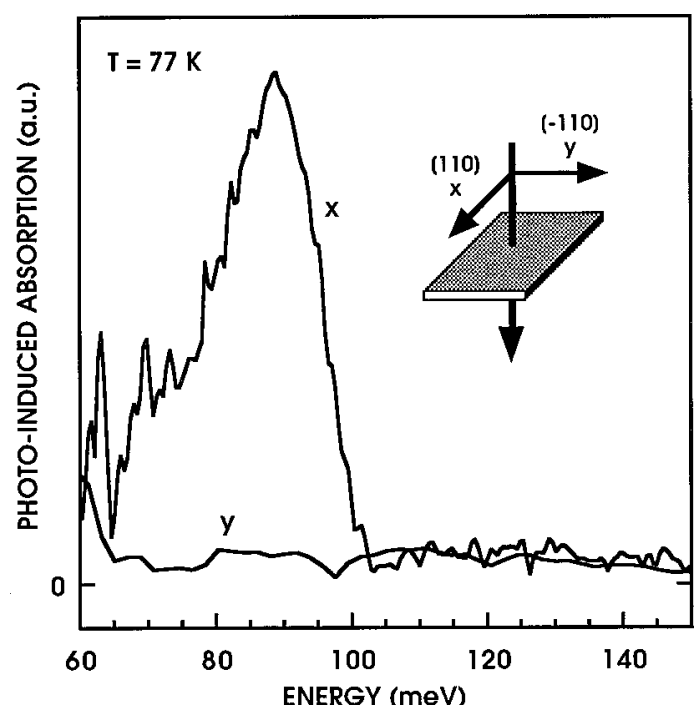

FIG. 2. $77 \mathrm{~K}$ photoinduced infrared absorption spectrum at normal incidence of the n.i.d. sample containing one plane of InAlAs/InAs clusters for light polarized along the [110] direction ( $x$ axis) and [ $\overline{1} 10]$ direction ( $y$ axis), respectively. Interband excitation at $4.5 \mathrm{~W} \mathrm{~cm}^{-2}$ is provided by an $\mathrm{Ar}^{++}$ laser.

at $77 \mathrm{~K}$ using a step-scan Fourier transform infrared (FTIR) spectrometer under optical excitation by an $\mathrm{Ar}^{++}$laser at a power density of $4.5 \mathrm{~W} \mathrm{~cm}^{-2}$. Figure 2 shows the photoinduced infrared absorption spectrum of sample A set at normal incidence for light polarized along the $x$ and $y$ axis, respectively. As seen, the $x$-polarized infrared spectrum reveals a narrow infrared resonance at $89 \mathrm{meV}(13.9 \mu \mathrm{m})$ with a full width at half maximum of $20 \mathrm{meV}$. The resonance vanishes when the polarization is rotated parallel to the $y$ axis. Separate experiments were carried out to measure the infrared transmission using a multipass waveguide configuration by alternately polishing at $45^{\circ}$ angle two opposite facets of the sample. Results confirm the existence of the $x$-polarized resonance. No resonances polarized along the $y$ axis or the $z$ axis are observed in the investigated $60-500$ $\mathrm{meV}$ energy range within the sensitivity of our measurements. Photoinduced measurements on the InP substrate do not show any resonance. These results confirm that the observed resonance is due to an intraband transition of the InAs/InAlAs dots.

In order to assess whether the intraband transition occurs in the valence band or in the conduction band, we have performed FTIR absorption measurements of the $p$-doped and $n$-doped samples at normal incidence. No resonance was observed for the $p$-doped sample in the investigated energy range. Figure 3 presents the absorption spectrum at 77 and $300 \mathrm{~K}$ in $x$ polarization divided by the spectrum in $y$ polarization of the $n$-doped samples $\mathrm{C}$ and $\mathrm{D}$, respectively. The infrared resonance occurs at $86 \mathrm{meV}(92 \mathrm{meV})$ for sample C (D). These observations clearly indicate that the absorption originates from an intraband transition in the conduction band of the InAs dots. The inset of Fig. 3 shows the integrated absorption of sample $\mathrm{C}$ at $300 \mathrm{~K}$ as a function of the light polarization angle, $\theta$, with $\theta=0^{\circ}\left(90^{\circ}\right)$ for light polarized along the $x(y)$ axis. The evolution is well fitted by a $\cos ^{2}(\theta)$ law. This strong polarization of the intraband absorption demonstrates that the excited state is confined along the [110] direction. The intraband resonance of sample C to AIP license or copyright, see http://ojps.aip.org/aplo/aplcr.jsp 


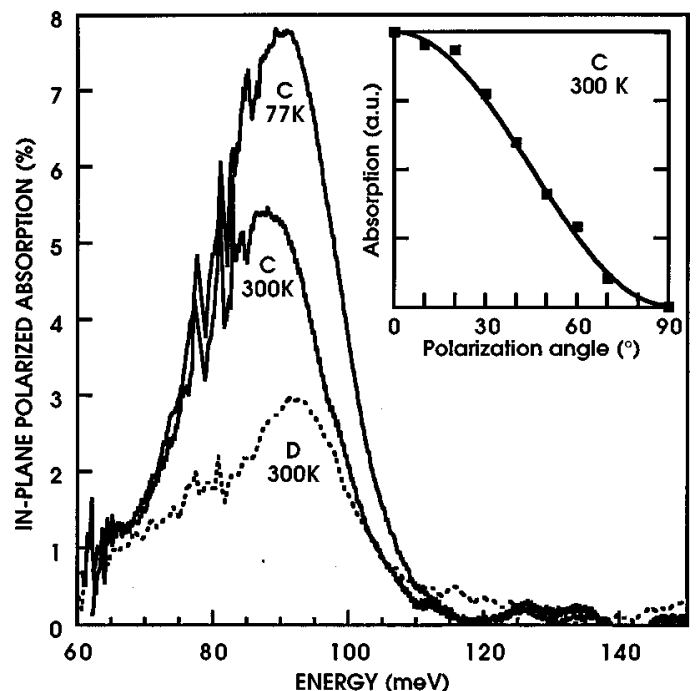

FIG. 3. Normal-incidence $x$-polarized absorption spectrum of the $n$-doped C (full curve) and D (dotted curve) samples at 77 and $300 \mathrm{~K}$. The spectra have been divided by the corresponding $y$-polarized spectra. The inset shows the polarization-angle dependence of the integrated infrared absorption of sample C. The $p$-doped sample has been used for measuring the background FTIR spectrum.

experiences a slight redshift of $3.2 \mathrm{meV}$ when the temperature is increased from 77 to $300 \mathrm{~K}$, which is typical of intraband transitions. ${ }^{12}$ The FWHM is of the order of $20 \pm 0.5$ $\mathrm{meV}$ at 77 and $300 \mathrm{~K}$. It is to be noticed that the linewidth is temperature independent within experimental accuracy. This result confirms that the linewidth is dominated by inhomogeneous broadening due to dot size fluctuations in agreement with the PLE results.

The absorption at maximum is of the order of $7.8 \%$ at 77 $\mathrm{K}$ for sample $\mathrm{C}$. It should be emphasized that this rather large value is achieved at normal incidence with a sample containing only ten planes of dots. The integrated absorption drops by $32 \%$ at room temperature. Assuming all impurities in the delta-doping layer are ionized and accounting for the measured absorption and FWHM, the equivalent absorption cross section at normal incidence for one plane of InAs dots is deduced to be of the order of $1.5 \times 10^{-14} \mathrm{~cm}^{-2}$. The average dipole length, $\langle x\rangle$, associated with the intraband transition is then estimated to be $21.3 \AA$. The oscillator strength, $f=\left(2 m_{0} E / h^{2}\right)|\langle x\rangle|^{2}$, where $E$ is the transition energy and $m_{0}$ the free electron mass, is of the order of 10.7 which is comparable to the value achieved for an intersubband transition in the conduction band of GaAs quantum wells ( $f$ $\approx 14)$. ${ }^{13}$

At this stage, it is not clear if the type of confinement in the elongated and aligned InAs dots is that of quantum wires or quantum dots. The shape of the aligned dots could be sufficiently modified by capping so that the dots be coupled in the [110] direction in the present samples. Assuming the shape and density of quantum dots measured by AFM, there should be an average number of 7 (3.5) electrons in each dot of sample C (D) based on their respective doping density. In the case of a quantum dot confinement one would expect the intraband absorption from the ground state to be the same for samples $\mathrm{C}$ and $\mathrm{D}$ because the ground state is already filled by 2 electrons with spin up and down. Results of Fig. 3 show that this is not the case and that the absorption grows by a factor 1.9 for sample $\mathrm{C}$ respective to sample D. Such behavior is expected for a quantum-wire confinement potential because there are always empty states above the Fermi energy in the ground subband. We have simulated the population difference between the upper and ground state assuming unidimensional subbands. ${ }^{14}$ Results show that the intraband absorption which is proportional to the population difference, drops by $30 \%$ when the temperature is increased from 77 to $300 \mathrm{~K}$ which is in agreement with experiments.

Note that the unidimensional density of states allows large quantum efficiencies of the normal-incidence intraband absorption to be achieved by adjusting the carrier concentration. In addition, electron capture times longer than in quantum wells can be expected because of the reduced dimensionality. ${ }^{15}$ We believe that the InAs/AlInAs/InP system may be of great interest for normal-incidence infrared photodetection applications.

The authors thank J.-P. Leburton for fruitful discussions.

${ }^{1}$ For a recent review, see D. Bimberg, M. Grundmann, and N. N. Ledenstov, MRS Bull. 23, 31 (1998), and references therein.

${ }^{2}$ H. Drexler, D. Leonard, W. Hansen, J. P. Kotthaus, and P. M. Petroff, Phys. Rev. Lett. 73, 2252 (1994).

${ }^{3}$ S. Sauvage, P. Boucaud, F. H. Julien, J. M. Gérard, and J.-Y. Marzin, J. Appl. Phys. 82, 3396 (1997).

${ }^{4}$ B. F. Levine, K. K. Choi, C. G. Bethea, and J. Malik, Appl. Phys. Lett. 50, 1092 (1987).

${ }^{5}$ J. Faist, F. Capasso, D. Sivco, C. Sirtori, A. L. Hutchinson, S. N. G. Chu, and A. Y. Cho, Science 264, 553 (1994); O. Gauthier-Lafaye, P. Boucaud, F. H. Julien, S. Sauvage, S. Cabaret, J.-M. Lourtioz, V. Thierry-Mieg, and R. Planel, Appl. Phys. Lett. 71, 3619 (1997).

${ }^{6}$ I. Gravé and Y. Yariv, in Intersubband Transitions in Quantum Wells, edited by E. Rosencher, B. Vinter, and B. Levine (Plenum, New York, 1992), p. 15.

${ }^{7}$ S. Pan, Y. P. Zeng, M. Y. Kong, J. Wu, Y. Q. Zhu, H. Zhang, J. M. Li, and C. Y. Wang, Electron. Lett. 32, 1726 (1996).

${ }^{8}$ K. W. Berryman, S. A. Lyon, and A. M. Segev, Appl. Phys. Lett. 70, 1861 (1997)

${ }^{9}$ J. Brault, M. Gendry, G. Grenet, G. Hollinger, Y. Desieres, and T. Benyattou, Appl. Phys. Lett. 73, 2932 (1998).

${ }^{10}$ J. M. Sallese, J. F. Carlin, M. Gaihanou, and M. Ilegems, Appl. Phys. Lett. 71, 2331 (1997).

${ }^{11}$ H. Lipsanen, M. Sopanen, and J. Ahopelto, Phys. Rev. B 51, 13868 (1995).

${ }^{12}$ P. von Allmen, M. Berz, G. Petrocelli, F.-K. Reinhart, and G. Harbeke, Semicond. Sci. Technol. 3, 1211 (1988).

${ }^{13}$ L. C. West and S. Eglash, Appl. Phys. Lett. 46, 1156 (1985).

${ }^{14}$ Y. Arakawa and H. Sakaki, Appl. Phys. Lett. 40, 939 (1982).

${ }^{15}$ S. Briggs and J.-P. Leburton, Phys. Rev. B 38, 8163 (1988). 